\title{
Effects of Flunixin Meglumine on Oxidant and Antioxidant System after Disbudding with Caustic Paste in Calves
}

\author{
Selvinaz Yakan ${ }^{1}$, Vesile Duzguner² \& Ozgur Aksoy³
}

\begin{abstract}
Background: Disbudding is often practiced in modern farm because of the reasons for the safekeeping of the animal, other animals lessen the risk of injury and less aggressive behavior. Without regard to the method of disbudding leads to disruption behavioral changes, cardiac and endocrine responses related pain of animals. Sedation, local anesthesia and analgesia are performed studies in order to eliminate of cardiac, endocrine, behavioral response which is caused by pain associated with disbudding. In this study, it was aimed to determine the effect on the oxidant and antioxidant system in the calves of disbudding by using the caustic paste with and without painkiller.

Materials, Methods \& Results: The animal material of the study was created 24 Simmentals calves in different sexes on average 2 weeks ( \pm 2 days) and $50 \mathrm{~kg}( \pm 15 \mathrm{~kg})$ live weight. The cases were randomly divided into 2 groups of 12 calves in each group. In the group I (analgesia group); caustic paste+painkiller $\left(2.2 \mathrm{mg}^{-1}\right.$ flunixin meglumine intravenous, IV) and group II (non-analgesia group); as disbudding applied with caustic pasta application only were divided into 2 groups. In the I group, 15th min before the application, flunixin meglumine at a dose of $2.2 \mathrm{mg}^{-1}$ was administered IV and the horn blunting was performed by caustic paste method. Blood samples were taken at 15,30 and 60th min after completion of horn blunting and physiological findings were recorded. In group II, the horns of the calves were disbudding with the same technique (but no analgesia aplication). Blood samples were taken at 15, 30 and 60th min after completion of horn blunting and physiological findings were record. Cortisol, glucose, total oxidant capacity (TOC) and total antioxidant capacity (TAC), 8-hydroxy-2'-deoxyguanosine (8-OHdG), glutathione (GSH), superoxide dismutase (SOD) enzymes measurements (ELISA) were performed in both groups. TOC and TAC tests were used to determine the oxidative stress index in the obtained serum, 8-OHdG measurement which is the most sensitive and most frequent oxidative DNA damage indicator for determination of oxidative damage; GSH, SOD enzymes were analyzed by ELISA using commercial kit for the evaluation of antioxidant potential. For evaluation of pain in animals, cortisol (ELISA) and glucose level in serum were measured with ELISA by using commercial kit. In the group without flunixin meglumine, cortisol was increased significantly from the 15th $\min (P<0.05)$, but there was no increase in the 30 and 60th min in painkiller administered group. Glucose levels were higher in the group not using painkillers than those used at 15th $(P<0.001)$ and 30, 60th min $(P<0.05)$. TOC $(P<$ $0.001)$ and $8-\mathrm{OHdG}(P<0.05)$ values were significantly higher in the group without painkiller while TAC, GSH and SOD levels $(P<0.05)$ were significantly decreased.

Discussion: It has been observed that sedation (such as xylazine) and local anesthesia (such as lidocaine) use removed pain-induced stress in the disbudding with caustic paste in calves. However, in order to remove the endocrine response, it is necessary to combine the anesthesia protocol with the analgesic (such as flunixin meglumine) combination. According to our results concluded that the painkiller given before the disbudding process was supporting the antioxidant system, reducing the level of cortisol and oxidative stress.
\end{abstract}

Keywords: Disbudding, flunixin meglumine, oxidant, antioxidant, calve. 


\section{INTRODUCTION}

Disbudding is often practiced in modern farm because of the reasons such as the reduction of carcass quality as a result of possible injuries, the less area it covers in the area of nutrition and during transporting, farm workers and other animals lessen the risk of injury and less aggressive behavior $[3,7,26]$. Three methods are used as thermal disbudding, surgical disbudding and caustic paste application for disbudding in young calves $[14,21,22]$. The application of caustic paste is disbudding process using a strong alkalinizing medication such as calcium hydroxide or sodium hydroxide [19,23,26]. Without regard to the method of disbudding leads to economic losses such as loss of weight, disruption of the physiology of animals with pain, and therefore behavioral changes, cardiac (heart rate) and endocrine (cortisol) responses. In particular, combinations of sedation and local anesthesia with analgesia are performed studies in order to eliminate of cardiac, endocrine, behavioral response lead to pain associated with disbudding $[1,6,20]$.

Sedation and local anesthetics decrease behaviors reactions associated with pain disbudding during (e.g., tail wagging, head movements, tripping, and rearing) and those findings of postoperative pain (rub head, shake head and ear flick) $[3,8,20]$.

Flunixin meglumine is a potent non-steroidal, anti-inflammatory, analgesia that pain relief in painful procedures by inhibiting the production of $\mathrm{PGE}_{2}$ from arachidonic acid $[4,12,16]$.

The aim of this study is to investigate the effect of antioxidant imbalance and the oxidative stress which may be caused by disbudding with the caustic paste of the applied anesthesia protocol and flunixin meglumine.

\section{MATERIALS AND METHODS}

\section{Methodology}

The animal selection of this study was performed at the commercial dairy farm, Ağrı, TR, 24 Simmentals calves in different sexes on average 2 weeks ( \pm 2 days) and $50 \mathrm{~kg}( \pm 15 \mathrm{~kg})$ live weight. The calves were randomly divided into 2 groups of 12 calves in each group, group I (analgesia group); caustic paste+painkiller $\left(2.2 \mathrm{mg}^{-1}\right.$ flunixin meglumine [Flunixamine $\left.{ }^{\circledR}\right]^{1}$ intravenous, IV) and group II (nonanalgesia group); as disbudding applied with caustic pasta application only were divided into 2 groups.
Groups

Group I (analgesia): Cases of caustic paste+painkiller group; the cases were included in the study, whose general examination was completed and decided to be healthy. Findings of respiratory rate, heart rate, and rectal temperature were recorded before doing anything to animals in this group. A $1.0 \times 32 \mathrm{~mm} \mathrm{IV}$ catheter was placed in the left Vena jugularis for blood sampling and drug administration. For biochemical analysis, $8 \mathrm{~mL}$ of blood was collected from the V. jugularis with vacuumed anticoagulant-free glass tubes. This time period was recorded at the 0th min in the study. At the same time, the animals were sedated with $0.2 \mathrm{mg}^{-1}$ IV Xylazine $\mathrm{HCl}\left(\text { Rompun }^{\circledR}\right)^{2}$. After 5 min sedation, flunixin meglumine $2.2 \mathrm{mg}^{-1}$ IV was administered. At the same time, $5 \mathrm{~mL}$ of Lidocaine $\mathrm{HCl}\left(\text { Jetokain }^{\circledR}\right)^{3}$ was applied to each horn region in order to anesthetize the corneal nerve. Disbudding was performed 15 min after sedation, local anesthesia, and painkiller application. The calve was prevented from moving firmly by an assistant for disbudding and hairs around the horn were uncovered well cut with a scissor. Then, vaseline is applied around the horn knob, the chemicals to be used have been prevented from flowing towards the skin and eye. Chemical substances to be used; it was calcium hydroxide rods in the form of round chalk (10 g REDFORT Boynuz Kalemi) ${ }^{4}$ specially prepared for this task. The tip of the caustic rod was pressed onto the horn knob with circular motion after slightly immersed on to water. The procedure was terminated when the horn knob was white first and then the bleeding indication was visible. This procedure was applied to each horn bud for about $35 \mathrm{~s}$. At the 15, 30 and 60th min of the first application (0th min), physiological parameters were recorded and, $8 \mathrm{~mL}$ blood samples were taken from the V. jugularis vacuumed anticoagulant-free glass tubes for biochemical analyzes.

Group II (non-analgesia): Cases of caustic paste only group; the same anesthetic protocol and disbudding were performed as in Group I. However, differently in this group, analgesia was not performed after sedation.

A single dose of Enrofloxacin (Baytril $^{\circledR) 2} 2.5$ $\mathrm{mg}^{-1} \mathrm{IV}$ was administered to all cases for prophylaxis. In addition, a skin ointment (Terramycin ointment) ${ }^{5}$ containing oxytetracycline was used on the wound resulting then disbudding in all cases.

\section{Biochemical analysis}

Blood samples were taken at $0,15,30$ and 60 th min of the applications then they were brought to the 
laboratory as soon as possible and centrifuged at 3000 rpm for $10 \mathrm{~min}$ at room temperature and kept at $-80^{\circ} \mathrm{C}$ until the samples were tested. Total oxidant capacity (TOC) [Bovine (TOC) ELISA kit] ${ }^{6}$ and total antioxidant capacity (TAC) [Bovine (TAC) ELISA kit $]^{6}$ tests were used to determine the oxidative stress indexin, 8-hydroxy-2'-deoxyguanosine (8-OHdG) [Bovine (8-OHdG) ELISA kit ${ }^{6}$ measurement which is the most sensitive and most frequent oxidative DNA damage indicator for determination of oxidative damage; Glutathione (GSH) [Glutathione Assay kit] ${ }^{7}$, Superoxide Dismutase (SOD) [Süperoksit Dismutase Assay kit] $]^{7}$ enzymes were analyzed by ELISA using commercial kit for the evaluation of antioxidant potential in the study. For evaluation of pain in animals, cortisol [Bovine (Cortisol) ELISA kit] ${ }^{6}$ and glucose [Glucose Colorimetric Assay Kit] ${ }^{7}$ level in serum were measured by using commercial kit.

\section{Statistical Analysis}

The one-way analysis of variance (ANOVA) and post hoc Duncan tests were performed on the data to examine the differences among groups using the SPSS statistical software package. The results are presented as average \pm SE. A value of $P<0.05$ was considered significant.

\section{RESULTS}

\section{Physiological findings}

There was no statistically significant difference in the findings of respiration rate in comparison within the group in both group I and group II at all times $(P>$ $0.05)$. There was no statistically significant difference in comparison between the groups in all time measurements $(P>0.05)$. In comparison within the group of heart rate, in the group I, the increase from the 0 th $\mathrm{min}$ to the 15 th min was statistically significant $(P<0.05)$. The changes that occurred at other measurement times were not statistically significant $(P>0.05)$. In group II, the increase from 0th min to 15th and 30th min was statistically significant $(P<0.05)$. The increase from 0 th to 60 th min was not statistically significant $(P>0.05)$. From the 15 th $\mathrm{min}$ the 30 and 60th-min decrease was statistically significant $(P<0.05) .30$ and 60th min were not statistically significant $(P>$ 0.05). There was no statistically significant difference in comparison between the groups at all measurement times $(P>0.05)$. Rectal temperature was found to be statistically significant in comparison to the within the group I, at the 0th min and at 15, 30 and 60th min $(P<0.05)$. No statistically significant difference was found between with 15 th min at 30 and 60th min of rectal temperature findings $(P>0.05)$. There was no statistically significant difference in the comparison of rectal temperature findings at 30 and 60th min $(P$ $>0.05)$. In Group II, there was no statistically significant difference in the results of all time measurements within the group $(P>0.05)$. The difference between the groups was statistically significant at 30th min $(P$ $<0.05)$. The measurement results of the physiological parameters are given in Table 1.

Table 1. Mean values and standard errors (Mean \pm SEM) of respiration rate, heart rate and rectal temperature at $0,15,30$ and 60 th min according to groups .

\begin{tabular}{|c|c|c|c|c|}
\hline $\begin{array}{l}\text { Time } \\
(\min )\end{array}$ & Group & $\begin{array}{l}\text { Respiratory rate } \\
\text { (Beat/min) }\end{array}$ & $\begin{array}{l}\text { Heart rate } \\
\text { (Pulse/min) }\end{array}$ & $\begin{array}{c}\text { Rectal temperature } \\
\left({ }^{0} \mathrm{C}\right)\end{array}$ \\
\hline \multirow{2}{*}{0} & I & $52.66 \pm 4.69$ & $150.00 \pm 8.18^{\mathrm{a}}$ & $38.81 \pm 0.08^{\mathrm{abc}}$ \\
\hline & II & $53.50 \pm 4.70$ & $152.83 \pm 4.34^{\mathrm{ab}}$ & $39.07 \pm 0.17$ \\
\hline \multirow{2}{*}{15} & I & $53.33 \pm 4.65$ & $182.91 \pm 11.9^{\mathrm{a}}$ & $39.08 \pm 0.08^{\mathrm{a}}$ \\
\hline & II & $54.33 \pm 3.69$ & $185.66 \pm 6.13^{\mathrm{abc}}$ & $39.25 \pm 0.10$ \\
\hline \multirow{2}{*}{30} & I & $53.66 \pm 4.57$ & $168.00 \pm 9.53$ & $39.04 \pm 0.06^{* a}$ \\
\hline & II & $50.00 \pm 4.70$ & $170.66 \pm 5.00^{\mathrm{ab}}$ & $39.33 \pm 0.11 *$ \\
\hline \multirow{2}{*}{60} & I & $45.66 \pm 4.10$ & $166.90 \pm 5.58$ & $39.09 \pm 0.06^{\mathrm{a}}$ \\
\hline & II & $50.16 \pm 3.82$ & $162.72 \pm 5.59^{b}$ & $39.09 \pm 0.10$ \\
\hline
\end{tabular}

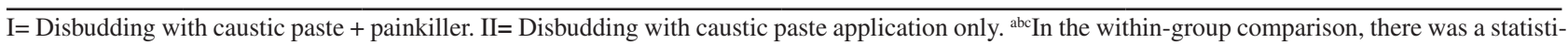
cally significant difference between the values of different letters in the same column $(P<0.05)$. *In the comparisons between groups, the measurements marked with asterisks at the same time period are considered statistically significant $(P<0.05)$. 


\section{Endocrine response}

A statistically significant difference was found between the measurement results with the 0 th min with the 15,30 and 60th min in Group I in the cortisol levels within-groups' comparison. Cortisol level decreased at 15 and 30th min according to pre-administration value and this decrease was statistically significant $(P<0.05)$. At the 60th min, the level of cortisol increased compared to the initial value and this increase was statistically significant $(P<0.05)$. There was no statistically significant difference between with 15th-min measurement results at 30 and 60th min on cortisol level $(P>0.05)$. The results of the measurements between 30 and 60th min were not statistically significant $(P>0.05)$.

In group II, an increase was observed between 15,30 and 60th min with the measurement results at 0 min and this increase was statistically significant $(P$ $<0.05)$. The measurement results at 15 and 30th min were statistically significant $(P<0.05)$. There was no statistically significant difference between the measurement results at 15 and 60th $\min (P>0.05)$. There was no statistically significant difference between the 30th min measurement results $(P>0.05)$. A statistically significant difference was found between all measurements time in the comparison between the groups. At 0 -min measurement results, cortisol level was lower in Group I and this differences were significant $(P<0.05)$. At 15, 30 and 60th min measurements, cortisol level

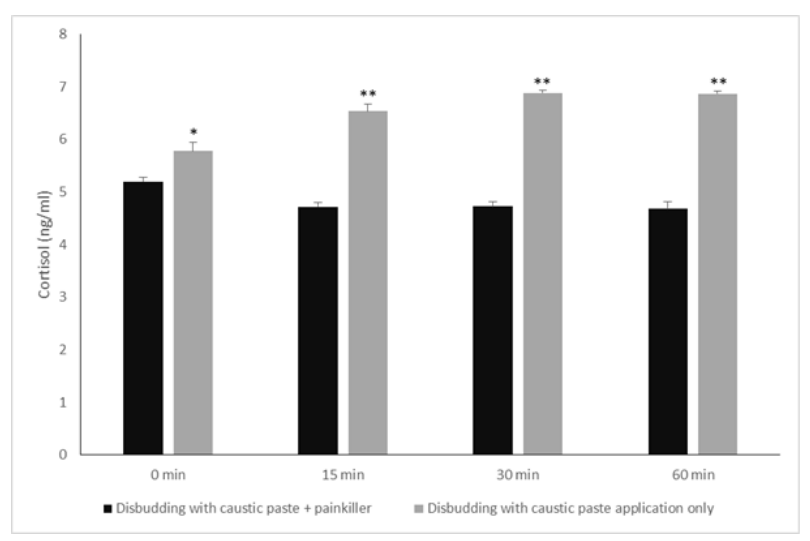

Graph 1. Changes in the cortisol level in group I and group II over time.

\section{Oxidant and antioxidant parameters}

In TOC measurements, the changes in the group I, between 0 and 15 th min were not statistically significant $(P>0.05)$. The increase from the 0 th $\mathrm{min}$ to the 30th and 60th min was statistically significant $(P<0.05)$. The increase with the 15 th $\min$ to 30 and was lower in Group I and the difference between the groups was statistically significant $(P<0.001)$. At all measurement times, cortisol level was lower in Group I than in Group II. The cortisol level measurement results of the groups are given in Graph 1.

In glucose levels within-groups' comparisons, the changes occurring in Group I at all measurement times were not statistically significant $(P>0.05)$. In group II, the level of glucose with 0 min increased at 15, 30 and 60th min and this increase was statistically significant $(P<0.05)$. There was no statistically significant difference between the 15 and 30th-min measurement results $(P>0.05)$. A statistically significant difference was found between the 15 and 60th min measurement results $(P<0.05)$. There was no statistically significant difference between the 30 and 60th-min measurement results $(P>0.05)$. In the comparison between the groups, the glucose level was measured at the higher level in the group I at the 0 -min measurement and this difference was found to be statistically significant $(P<0.05)$. At the 15 th $\mathrm{min}$, the glucose level increased in Group II and this increase was statistically significant $(P<0.001)$. At 30 and 60th min, glucose level was lower in group I and statistically, a significant difference was found between groups $(P<$ $0.05)$. From the 15 th min when disbudding was made, glucose level was lower in group I than in group II. The glucose level measurement results of the groups are given in Graph 2.

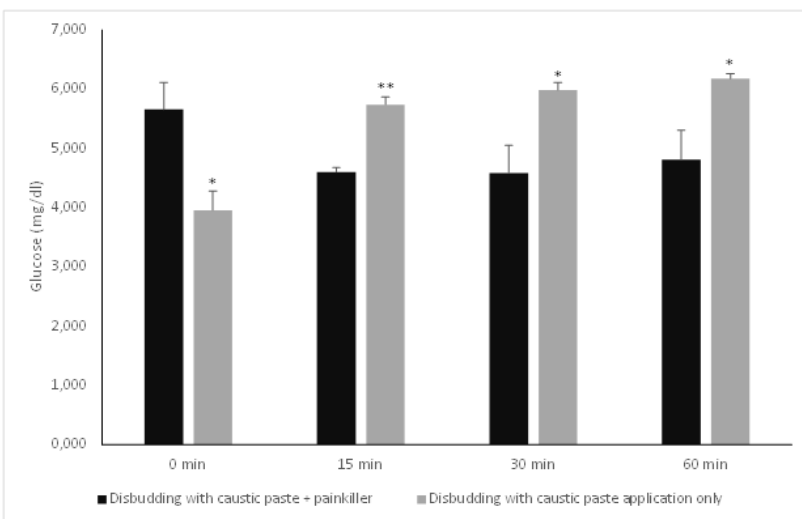

Graph 2. Changes in the glucose level in group I and group II over time.

60th min was statistically significant $(P<0.05) .30$ and 60th min were not statistically significant $(P>0.05)$.

In Group II within-group comparison, the increase in the values at $0 \mathrm{~min}$ and at all measurement times was statistically significant $(P<0.05)$. There was no statistically significant difference between the 
groups in the measurements at $0 \min (P>0.05)$. A statistically significant difference was found between measurements at 15 and 30th $\min (P<0.05)$ and the difference between 60th-min measurement results was statistically significant $(P<0.001)$. In Group II, the increase in total oxidant capacity was higher.

There was no statistically significant difference in TAC measurements of within-group comparison both Group I and Group II at all measurement times $(P$ $>0.05)$. There was a statistically significant difference between all sampling times in the comparison between the groups $(P<0.05)$. Total antioxidant capacity measurements were higher in Group I.

The results of 8-OHdG were statistically significant in Group I, between with 0 min, at 15, 30 and 60th $\min (P<0.05)$. The measurement results with 15 th min, 30 and 60th min were statistically significant $(P<0.05)$. The results of the measurements at 30 and 60th min were not statistically significant $(P>0.05)$. In group II, the measurement results with $0 \mathrm{~min}, 15,30$ and 60th $\mathrm{min}$ were statistically significant $(P<0.05)$. The measurement results with 15 th $\mathrm{min}, 30$ and 60 th min were statistically significant $(P<0.05)$. The results of the measurements at 30 to 60 th min were not statistically significant $(P>$
0.05). There was no statistically significant difference in comparison between the groups in the measurement results at $\min (P>0.05)$. There was a statistically significant difference between the measurement results at 15, 30 and 60th $\min (P<0.001)$. The level of 8-OHdG is lower in Group I.

There was no statistically significant difference in GSH measurement within-group comparison both Group I and II at all sampling times $(P>0.05)$. There was no statistically significant difference in the 0 -min measurement results between the groups $(P>0.05)$. A statistically significant difference was found between 15 and 30th $\min (P<0.05)$ and 60th $\min (P<0.001)$. GSH level increased more in Group I according to initial value.

There was no statistically significant difference in SOD measurement within-group comparison both Group I and II during all sampling times $(P>0.05)$. There was no statistically significant difference in the 0 -min measurement results between the groups $(P>$ $0.05)$. A statistically significant difference was found between 15, 30 and 60th min $(P<0.05)$. SOD level was higher in Group I. TOC and TAC measurements, 8-OHdG, GSH, SOD of the groups' measurement results are given in Table 2.

Table 2. Mean values and standard errors (Mean \pm SEM) of biochemical findings at $0,15,30$ and 60 th min according to groups.

\begin{tabular}{|c|c|c|c|c|c|c|}
\hline $\begin{array}{l}\text { Time } \\
(\min )\end{array}$ & Group & $\begin{array}{c}\text { TOC } \\
(\mu \mathrm{mol} / \mathrm{L})\end{array}$ & $\begin{array}{c}\text { TAC } \\
(\mathrm{U} / \mathrm{mL})\end{array}$ & $\begin{array}{l}\text { 8-OHdG } \\
(\mathrm{ng} / \mathrm{mL})\end{array}$ & $\begin{array}{c}\text { GSH } \\
(\mathrm{mmol} / \mathrm{L})\end{array}$ & $\begin{array}{c}\text { SOD } \\
(\mathrm{U} / \mathrm{mL})\end{array}$ \\
\hline \multirow{2}{*}{0} & I & $32.50 \pm 2.78^{\mathrm{ac}}$ & $2.73 \pm 0.39 *$ & $3.82 \pm 0.26^{\mathrm{bc}}$ & $292.83 \pm 2.76$ & $7.26 \pm 0.34$ \\
\hline & II & $30.00 \pm 3.01^{\mathrm{abc}}$ & $2.13 \pm 0.17 *$ & $3.86 \pm 0.28^{\mathrm{bc}}$ & $303.50 \pm 3.16$ & $6.90 \pm 0.58$ \\
\hline \multirow{2}{*}{15} & I & $40.00 \pm 1.74^{* b c}$ & $2.80 \pm 0.17 *$ & $3.76 \pm 0.23 * * a b c$ & $316.00 \pm 2.76 *$ & $8.69 \pm 1.01 *$ \\
\hline & II & $52.50 \pm 3.50 * a b c$ & $2.12 \pm 0.17 *$ & $4.58 \pm 0.43^{* * a b c}$ & $307.00 \pm 3.37 *$ & $6.18 \pm 0.40 *$ \\
\hline \multirow{2}{*}{30} & I & $55.00 \pm 3.79 * a b$ & $2.95 \pm 0.14 *$ & $4.07 \pm 0.008 * * a b$ & $318.27 \pm 2.22^{*}$ & $9.13 \pm 0.59 *$ \\
\hline & II & $75.00 \pm 4.14^{* a b c}$ & $2.12 \pm 0.23 *$ & $4.24 \pm 0.006^{* * a b}$ & $304.45 \pm 4.39 *$ & $7.00 \pm 0.41 *$ \\
\hline \multirow{2}{*}{60} & I & $63.33 \pm 3.09 * * a b$ & $3.06 \pm 0.13^{*}$ & $4.03 \pm 0.009 * * a b$ & $322.83 \pm 3.57 * *$ & $9.06 \pm 0.55 *$ \\
\hline & II & $94.16 \pm 4.51 * * a b c$ & $1.85 \pm 0.35^{*}$ & $4.16 \pm 0.009 * * a b$ & $307.41 \pm 2.22 * *$ & $6.69 \pm 0.38 *$ \\
\hline
\end{tabular}

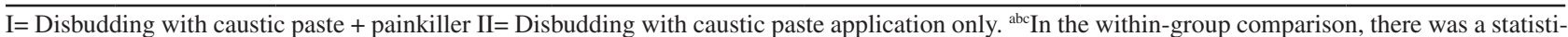
cally significant difference between the values of different letters in the same column $(P<0.05)$. *In the comparisons between groups, the measurements marked with asterisks at the same time period are considered statistically significant $(P<0.05)$. $* P<0.05 ; * * P<0.001$.

\section{DISCUSSION}

Disbudding, when considering the economic contribution that it can bring to the farm is an application that needs to be done [23]. Disbudding is a stressful process on cattle, which results in more stress in adult cattle than in younger cattle and the effect of horn breaking in elderly cattle continues for a longer time
$[14,20]$. As the horn grows, the healing period of the wound, which is opened at the site of the horn which is to be cut out, is also extended and the animal is more affected. For all these reasons, it is of great benefit to make disbudding in the cattle as early as possible, both in terms of ease of breeding and less influence on the animal [20]. 
Having in mind calve welfare, the disbudding should be performed during the first weeks of life, and the final date to perform this procedure is an age of eight weeks [5,20,22]. Disbudding is a painful and difficult procedure for the calve and it is necessary to minimize this stress. In addition, the method used to disbudding is another important factor determining degree influence of animal [5,14]. The easiest and least painful method of disbudding is the prevention of the horn out of chemical substances [25]. The use of caustic paste application with disbudding is recommended for use on calves between 8-14 days [14].

The physiological parameters of respiration rate, heart rate, and cornea temperature are evaluated as the stressor of pain sensation caused by the disbudding process $[2,6,14]$. In researches, respiratory rate, cardiac frequency findings vary according to the method used, the combination of anesthesia protocol and analgesia applied [10]. Alvarez et al. [2], in the goats where they applied disbudding by the cauterization method, it is reported that cardiac and respiratory numbers are similar in the groups that did and did not receive local anesthesia [10].

In disbudding methods, it is reported that heart and respiratory numbers are increased due to pain. In their study, they used the cauterization method to perform disbudding in calves, it is reported that the findings of heart and respiration are closer to the physiological limits in local anesthesia + meloxicam groups than only those who receive local anesthesia. According to Huber et al. [11], there is no difference between the groups of control and local anesthesia, flunixin meglumine, local anesthesia + flunixin meglumine groups in the calves where they applied disbudding with electrocautery. The frequency of the heart increases very clearly in the disbudding process, especially when anesthesia is not applied [14]. In the study of Yanmaz et al. [26], operation and cauterization methods, and there was no statistical change in the number of respirations in the untreated groups when analgesia was applied to the calves on which the disbudding. In our study, cases were divided into two groups as sedation + local anesthesia + analgesia (group I) and sedation + local anesthesia (group II) that calves applied to disbud with the caustic paste application. Changes in respiratory rate in both groups were observed at physiological limits. The heart rate was increased according to the initial value in both groups at the 15th min when disbudding. We think that this increase is a sign of acute stress. After 15 min in both groups, heart rate findings were viewed approaching the initial value. Also in our study, which we evaluated for rectal temperature findings, an increase in rectal temperature findings were observed in the analgesia group compared to the baseline value. However, this increase was statistically significant with physiological limitations and no medical treatment was required. In the group without analgesia, there was no statistically change in rectal temperature findings for $60 \mathrm{~min}$. We think because of using the anesthesia protocol and the method to monitor in the reference intervals of physiological parameters changes during the experiment. Furthermore, no significant findings were found to record the behavioral response. Our findings were supporting to knowledge, xylazine does not affect the initial cortisol response that reflects the pain in the disbudding process but removes the behavioral response with the calming effect during disbudding [20].

It is reported that immediately after disbudding, the increased cortisol level reflects pain-induced stress and to eliminate of the cortisol response of the applied local anesthesia. NSAIDs administered intravenously before 15-20 min from disbudding do not only remove pain, but also contribute to healing mechanisms and inflammation in the horn region during disbudding $[20,26]$. In research by Stafford and Mellor [20], have been reported that to eliminate of the cortisol response by the anesthesia of the cornual nerve block made with lidocaine 15-20 min before the disbudding by amputation method and cortisol level is close to the initial value within $2 \mathrm{~h}$ after the disbudding. Administration of a local analgesic decreased the baseline increase in plasma cortisol level [3]. Numerous studies point out that cortisol levels have been reported to suddenly increase after disbudding and remain at high levels for 4-5 h [2,6,12,14,24,26]. In study of Stafford and Mellor [20], has been reported that prevent the increase of cortisol level and use of non-steroidal anti-inflammatory ketoprofen also local anesthesia to the horn region in order to anesthetize of cornual nerve and the xylazine in disbudding with cauterization. One research showed that providing non-steroidal anti-inflammatory drugs, in addition to a local anesthetic, can keep plasma cortisol to initial levels for as long as $6 \mathrm{~h}$ after dehorning [17]. In the 
study by Huber et al. [11] are similar to reported of levels cortisol in the untreated control group and use of flunixin meglumine in calves applied to disbudding with cauterization. In our study unlike the results of the researchers $[2,6,12,14,24,26]$, of cortisol level was observed in lower levels in to applied group flunixin meglumine. The lower of determination of serum cortisol levels compared to those not administered in analgesia-applied in calves confirms reduced the paininduced stress of flunixin meglumine [20]. Stress and inflammation lead to excessive glucose production and the degree of hyperglycemia increases as the severity of the illness or injury increases. Serum glucose levels in calves treated with analgesics in both operation and cauterization groups were lower than those without analgesics [26]. In our study, serum glucose level was lower in the analgesia group similar to the findings previously described [26].

In study to assessment of pain-related stress in disbudding methods, behavioral findings so, the reactions to pain of animals, evaluation of physiological parameters; heart rate and respiration, cornual temperature, rectum temperature, endocrine response; cortisol, glucose level as well as of such as findings; on the antioxidant defense system have gained importance investigation of the effects of non-steroidal anti-inflammatory of analgesia agents used to and the applied anesthesia protocol and oxidative stress induced by disbudding methods.

Many molecules and methods have been developed for evaluating oxidative stress. Several studies report that the TOC and TAC parameters may be useful noninvasive markers for oxidative stress-related diseases. For this reason, in recent years it has been suggested to measure TOC, TAC to determine the net oxidant and antioxidant status and their equilibrium [15]. In our study, TOC measurements decreased from the 15 th min in the analgesia group but increased in the group without analgesia. TAC measurements were found to be higher in the analgesia group than in the untreated group. Oxidative stress is known to cause damage to a number of lesions such as base and sugar modifications on DNA, single and double chain breaks, basic regions, DNA-Protein cross-linking with different mechanisms. One of the most sensitive markers of this oxidative damage in DNA is 8-OHdG [27]. In our study, analgesia was not statistically significant in the group applied, but it was lower than in the group without analgesia.

Glutathione in the antioxidant defense system, which plays a role in effectively and safely eliminating the reactive oxygen species formed in the living organism, acts as nonenzymatic and SOD enzymatic antioxidant. GSH is an important endogenous antioxidant with numerous cellular functions that protect against harmful effects of free radicals and reactive oxygen species. SOD is the first enzyme in the antioxidative system and increases in inflammation and pain $[9,18]$. In our study, GSH and SOD levels were found to be at lower levels in the non-analgesic group, leads to the stress is not significant disbudding method and of disbudding must be combined with the combination of anesthesia and analgesia.

\section{CONCLUSION}

As a result, it has been observed that sedation (such as xylazine) and local anesthesia (such as lidocaine) use removed pain-induced stress in the disbudding with caustic paste in calves. However, in order to remove the endocrine response, it is necessary to combine the anesthesia protocol with the analgesic (such as flunixin meglumine) combination. According to our results concluded that the painkiller given before the disbudding process was supporting the antioxidant system, reducing the level of cortisol and oxidative stress.

\section{MANUFACTURERS}

${ }^{1}$ Zoetis Türkiye. Istanbul, Turkey.

${ }^{2}$ Bayer Türk Kimya San. Tic. Ltd. Şti. Istanbul, Turkey.

${ }^{3}$ Adeka İlaç Sanayi ve Ticaret A.Ş. Samsun, Turkey.

${ }^{4}$ Seçkim Medikal Ürünleri Ltd. Şti. Izmir, Turkey.

${ }^{5}$ Pfizer Türkiye. Istanbul, Turkey.

${ }^{6}$ Shanghai Sunred Biological Technology. Shangai, China.

${ }^{7}$ Cayman Medical Company. Ann Arbor, MI, USA.

Acknowledgements. This research was supported by Ardahan University Scientific Research Projects Unit by 2017/002 number project. The authors wish to thank Haliloğulları Dairy Farm, Ağrı, TR.

Ethical approval. The study was approved by the animal local Ethitcs Committe (KAÜ, HAYDEK Date: 06.07.2017. Number: 2017-064), Kafkas University.

Decleration of interest. The authors report no conflicts of interest. The authors alone are responsible for the content and writing of the paper. 


\section{REFERENCES}

1 Akın I. \& Karademir U. 2017. Comparison of the efficacies of meloxicam and flunixin meglumine on some haemostatic variables in dehorned Holstein heifers. Journal of Faculty Veterinary Medicine, Erciyes University. 14(2): 87-91.

2 Alvarez L., Nava R.A., Ramirez A., Ramirez E. \& Gutierrez J. 2009. Physiological an behavioural alterations in disbudded goat kids with and without local anesthesia. Applied Animal Behaviour Science. 117(3/4): 190-196.

3 Faulkner P.M. \& Weary D.M. 2000. Reducing pain after dehorning in dairy calves. Journal of Dairy Science. 83: 2037-2041.

4 Fraccaro E., Coetzee J.F., Odore R., Edwards-Callaway L.N., Kukanich B., Badino P., Bertolotti L., Glynn H., Dockweiler J., Allen K. \& Bergamasco L. 2013. A study to compare circulating flunixin, meloxicam and gabapentin concentrations with prostaglandin $\mathrm{E}(2)$ levels in calves undergoing dehorning. Reserach in Veterinary Science. 95 : 204-211.

5 Goonewardene L.A., Price M.A., Okine E. \& Berg R.T. 1999. Behavioral responses to handling and restraint in dehorned and polled cattle. Applied Animal Behaviour Science. 64: 159-167.

6 Gorgul O.S., Yasar N.G., Kanık S., Salcı H. \& Altıkardesler A. 2002. Evalation of endocrine, cardiac responses and operation technique in cattle undergoing cosmetic dehorning under local anaesthesia with and without sedation. Veteriner Cerrahi Dergisi. 8: 5-10.

7 Gottardo F., Nalon E., Contiero B., Normando S., Dalvit P. \& Cozzi G. 2011. The dehorning of dairy calves: Practices and opinions of 639 farmers. Journal of Dairy Science. 94: 5724-5734.

8 Grondahl-Nielsen C., Simonsen H.B., Lund J.D. \& Hesselholt H. 1999. Behavioural, endocrine and cardiac responses in young calves undergoing dehorning without and with use of sedation and analgesia. The Veterinary Journal. 158(1): 1-3.

9 Gurgoze S.Y., Sindak N., Yılmaz S., Sertkaya H. \& Ozan S.T. 2003. The effects of corticosteroid treatment of some antioxidant enzymes and the level of lipid peroxidation in cattles with burcitis precarpalis. Van Veterinary Journal. 14: 97-101.

10 Heinrich A., Duffield T.F., Lissemore K.D., Squires E.J. \& Millman S.T. 2009. The impact of meloxicam on postsurgical stress associated with cautery dehorning. Jornal of Dairy Science. 92: 540-547.

11 Huber J., Amholdt T., Mostl E., Gelfert C.C. \& Drillich M. 2013. Pain management with flunixin meglumine at dehorning of calves. Journal of Dairy Science. 96(1): 132-140.

12 Korkmaz M., Saritas Z.K., Bulbul A. \& Demirkan I. 2015. Effect of pre-emptive dexketoprofen trometamol on acute cortisol inflammatory response and oxidative stress to hoironson disbudding in calves. Journal of the Faculty Veterinary Medicine, Kafkas University. 21: 563-568.

13 Kukanich B., Bidgood T. \& Knesl O. 2012. Clinical pharmacology of non-steroidal anti-inflammatory drugs in dogs. Veterinary Anaesthesia and Analgesia. 39: 69-90.

14 Kupezynski R., Budny A., Spitalniak K. \& Traez E. 2014. Dehorning of calves- methods of pain and stress alleviationReviewed. Annals of Animal Science. 14: 231-243.

15 Kurku H. \& Soran M. 2017. Oxidative stress levels of serum and urine in enuresis. Van Medical Journal. 24(4): 267271.

16 Mathews K.A. 2002. Non-steroidal anti-inflammatory analgesics: reviewev of current practice. Journal of Veterinary Emergency Critical Care. 12(2): 89-97.

17 McMeekan C., Stafford K.J., Mellor D.J., Bruce R.A., Ward R.N. \& Gregory N.G. 1999. Effects of a local anaesthetic and a nonsteroidal anti-inflammatory analgesic on the behavioral responses of calves to dehorning. New Zealand Veterinary Journal. 47: 92-96.

18 Meral O., Ercan N. \& Fidancı U.R. 2017. Lipid peroxidation level and antioxidant enzyme activities in septicemic calves. Veterinary Journal of Ankara University. 64: 161-164.

19 Neely C.D., Thomson D.U., Kerry C.A. \& Reinhardt C.D. 2014. Effects of three dehorning techniques on behavior and wound healing in feedlot cattle. Journal of Animal Science. 92(5): 2225-2229.

20 Stafford K.J. \& Mellor D.J. 2004. Dehorning and disbudding distress and its allevation in calves. The Veterinary Journal. 169: 337-349.

21 Stilwell G., Carvalho R.C., Lima M.S. \& Broom D.M. 2009. Effect of caustic paste disbudding, using local anaesthesia with and without analgesia, on behaviour and cortisol of calves. Applied Animal Behaviour Science. 116: 35-44. 
22 Stilwell G., Carvalho R.C., Carolino N., Lima M.S. \& Broom D.M. 2010. Effects of hot-iron disbudding behaviour and plasma cortisol of calves sedated with xylazine. Research in Veterinary Science. 88: 188-193.

23 Stilwell G., Lima M.S., Carvalho R.C. \& Broom D.M. 2012. Effects of hot-iron disbudding, using region anesthesia with and without carprofen, on cortisol an behaviorur of calves. Reserch in Veterinary Science. 92: 338-341.

24 Sylvester S.P., Stafford K.J., Mellor D.J., Bruce R.A. \& Ward R.N. 2004. Behavioural responses of calves to amputation dehorning with and without local anesthesia. Australian Veterinary Journal. 82(11): 697-700.

25 Vickers K.J., Niel L., Kiehlbauch L.M. \& Weary D.M. 2005. Calf response to caustic paste and hot-iron dehorning using sedation with and without local anesthetic. Journal Dairy Science. 88: 1454-1459.

26 Yanmaz L.E., Kaya M., Dogan E., Okumus Z. \& Kaynar O. 2015. Assessment of the pain level depends on the disbudding techniques and analgesic drug administration in calves. Harran University Journal of the Faculty of Veterinary Medicine. 4(1): 22-26.

27 Yokus B. \& Cakir D.U. 2002. Biomarker of in vivo oxidative DNA damage 8-hydroxy-2'-deoxyguanosine. Türkiye Klinikleri Journal of Medical Sciences. 22(5): 535-543. 